\title{
Britain grappling with dementia prevalence
}

$\mathrm{W}$

ith as many as 1.7 million Brits projected to be diagnosed with dementia by 2051, and the average cost of caring for those afflicted pegged at roughly $£ 25000$ a year, it's no surprise the British government has unveiled a 5year dementia strategy that proposes to place a "memory clinic" in every town.

Yet skeptics aren't convinced that the government's strategy is an appropriate response and argue that the monies — to start, $£ 150$ million over 2 years - might be better spent on enhancing primary care by physicians. Still others, including the United Kingdom's National Institute for Health and Clinical Excellence, have urged a focus on treatment, arguing that there's a regional discrepancy in the availability of memory drugs and that treatment should be constrained to those with more advanced symptoms and not those in the early stages of dementia.

The government, though, believes it can mitigate the effects of the dementia crisis through its new strategy, which includes plans to train general practitioners to spot early signs of dementia in patients, hire more dementia specialists in hospitals and care homes, as well as introduce a public awareness program to help offset the stigma associated with the illness.

A 2007 report from the National Audit Office indicated that only $31 \%$ of general practitioners in England felt they had enough training to diagnose and manage the disease themselves.

That is likely one of the reasons why only one-third of dementia sufferers receive a formal diagnosis, says Roy Jones, professor of the British Institute for Care of the Elderly. "Ideally, there would be screening in every town and then referral to a specialized clinic. Diagnosing dementia in its early stages is not easy, for there are other conditions, such as depression and thyroid problems, which can cause similar symptoms and need to be ruled out."

Even after diagnosis, the precise nature of the dementia - whether Alzheimer disease, vascular dementia or another form - must be ascertained before treatment can commence. Compounding the problem is that existing drug therapies for mild to moderatestage Alzheimer sufferers appear to be effective in but $50 \%$ of patients.

Although it's often argued that early diagnosis is moot because there's limited evidence that early treatment has an impact, Jones dismisses that as a "nihilistic mentality." For example, the National Institute for Health and Clinical Excellence limitation on patient access to existing Alzheimer drugs such as cholinesterase inhibitors on the grounds that they are not cost effective during early stages of the disease, fails to take into account the cost of residential care or the burden on family caregivers, Jones says.

Rebecca Wood, chief executive officer of the Alzheimer's Research Trust,

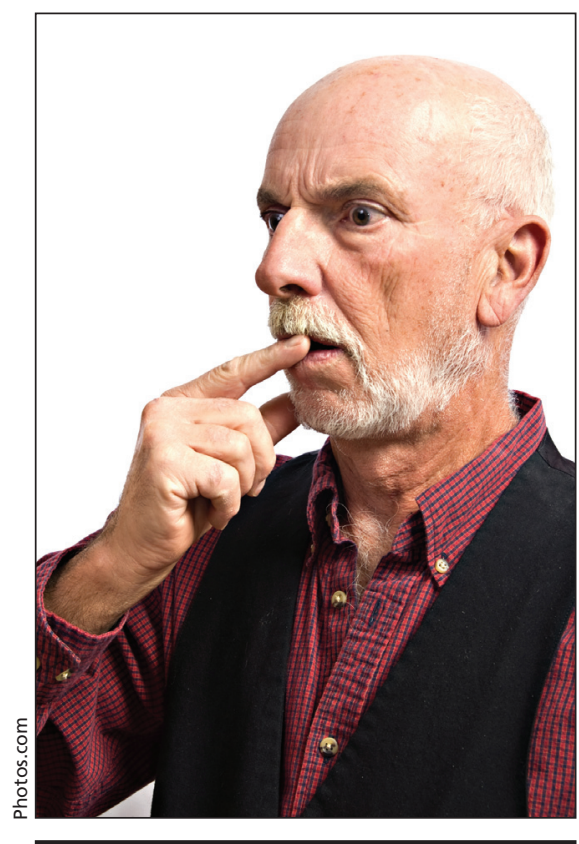

Experts have warned that Britain is heading for a dementia "epidemic," as a consequence of the nation's bingedrinking culture and the aging of baby boomers.

says the current cost of providing medications to dementia patients is about $£ 2.50$ a day, while nowhere near adequate amounts of money are being spent on dementia research in light of the incidence of the illness. "When we begin to look at how our population is aging, the figures are scary." - Mary Helen Spooner, London, England

DOI:10.1503/cmaj.090406 